\title{
Numb localization nailed
}

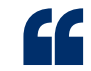

...cascade of events that regulate Numb asymmetry...
The Drosophila melanogaster Numb protein is uniformly distributed on the cortex and in the cytoplasm of neural precursor cells in interphase, but shows a polarized localization during mitosis. This results in the asymmetric segregation of Numb into one of the two daughter cells and results in distinct cell fates. How the unusual asymmetric localization of Numb in mitosis is achieved has been a mystery for many years. But Juergen Knoblich and colleagues have now worked out the mechanistic details.

Aurora A (AurA, also known as AUR) is a mitotic kinase that is required for Numb asymmetry; indeed, Numb is mislocalized in aurA mutants. Numb is also mislocalized in cells that express a nonphosphorylatable mutant of lethal (2) giant larvae (LGL), which is a substrate for atypical protein kinase $\mathrm{C}$ (aPKC) - a protein of the partitioning defective (PAR) complex. The levels of phosphorylated LGL and of aPKC activity are reduced in aurA mutants. The authors demonstrate that AurA phosphorylates PAR6 in the PAR complex. Phosphorylation of PAR6 interferes with its interaction with aPKC. So, given that PAR6 is an inhibitor of aPKC activity, the authors suggest that AurA releases its inhibition by dissociating aPKC from PAR6, resulting in phosphorylation of LGL.

In interphase, LGL localizes to the cell cortex, where it associates with PAR6 and aPKC. At the onset of mitosis, phosphorylation of LGL by aPKC causes its release from the posterior cortex (where aPKC is localized) and gives rise to an asymmetric anterior localization.
But how do these findings relate to Numb asymmetry?

The next step in the cascade of events that regulate Numb asymmetry involves the PAR subunit Bazooka (BAZ). Knoblich and coworkers showed that BAZ functions downstream of LGL and that BAZ fails to localize to the posterior cortex in aurA mutants. Because aurA mutants also fail to release LGL from the posterior cortex, this implies that LGL inhibits the cortical localization of BAZ. LGL and BAZ compete for interaction with the PAR components PAR6 and aPKC, and form alternative PAR complexes. aurA mutant cells contain excess levels of LGL-containing complex compared with BAZ-containing complex.

How does BAZ regulate Numb asymmetry? Numb is a substrate for aPKC, and its phosphorylation releases Numb from the cell cortex. The authors showed that BAZ changes the substrate specificity of aPKC; the BAZ complex phosphorylates Numb, whereas the LGL complex does not. So, the exchange of LGL for BAZ in the PAR complex leads to the phosphorylation of Numb by aPKC on one side of the cell cortex, thereby limiting the presence of Numb to the opposite side - ultimately leading to asymmetric cell division.

Arianne Heinrichs

ORIGINAL RESEARCH PAPER Wirtz-Peitz, F. et al. Linking cell cycle to asymmetric division: Aurora-A phosphorylates the Par complex to regulate Numb localization. Cell 135, 161-173 (2008)

FURTHER READING Gonczy, P. Mechanisms of asymmetric cell division: flies and worms pave the way. Nature Rev. Mol. Cell Biol. 9, 355-366 (2008)
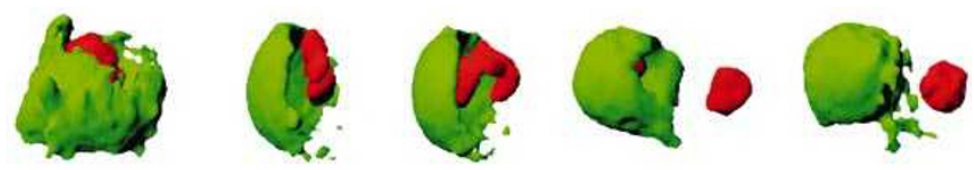

Images represent three-dimensional reconstructions of a Drosophila melanogaster sensory organ precursor cell in (from left to right) interphase, metaphase, early and late anaphase and telophase of asymmetric cell division. Segregation of chromatin (red) is symmetric, whereas Numb (green, visualized by the adaptor protein PON fused to green fluorescent protein segregates asymmetrically. Image courtesy of J. Knoblich, Vienna, Austria. 